\title{
Politopo baseado em distâncias para o problema clássico de coloração de vértices em grafos
}

\author{
Bruno Dias $^{1}$, Rosiane de Freitas ${ }^{1}$, Javier Marenco $^{2}$, Nelson Maculan ${ }^{3}$ \\ ${ }^{1}$ Instituto de Computação - Universidade Federal do Amazonas (UFAM) \\ Av. Rodrigo Otávio, 3000 - 69000-000 - Manaus - AM - Brasil \\ ${ }^{2}$ Departamento de Computación - Universidad de Buenos Aires (UBA) \\ Buenos Aires - Argentina \\ ${ }^{3}$ PESC/COPPE - Universidade Federal do Rio de Janeiro (UFRJ) \\ Caixa Postal 68530 - 21945-970 - Rio de Janeiro - RJ - Brasil \\ \{bruno.dias, rosiane\}@icomp.ufam.edu.br, jmarenco@dc.uba.ar, \\ maculanecos.ufrj.br
}

\begin{abstract}
In this work, we present a distance-based model for the classic vertex coloring problem in graphs (VCP). The integer linear programming formulation uses decision variables representing the distance between the colors assigned to every pair of distinct vertices instead of explicitly providing the colors assigned to each vertex. We show close relations between this formulation and the socalled orientation model for VCP, also proposed by the authors of this work. In particular, we prove that we can translate many facet-inducing inequalities for the orientation model polytope into facet-inducing inequalities for the distance model polytope, and vice versa.
\end{abstract}

Resumo. Neste trabalho, apresenta-se o modelo baseado em distâncias para o problema clássico de coloração de vértices em grafos (VCP). A formulação de programação linear inteira utiliza variáveis de decisão que representam a distância entre cores atribuídas para cada par de vértices distintos, no lugar de fornecer explicitamente tais cores. Mostra-se que há uma relação próxima entre esta formulação e o modelo baseado em orientação para o VCP, proposto também pelos autores deste trabalho. Em particular, prova-se que desigualdades indutoras de facetas para o modelo baseado em orientação podem ser traduzidas em desigualdades indutoras de facetas para o modelo baseado em distâncias e vice-versa.

\section{Introdução}

O problema clássico de coloração de vértices em grafos (em inglês, vertex coloring problem - VCP) consiste em, para um grafo simples não direcionado $G=(V, E)$, encontrar um mapeamento $c: V \rightarrow \mathbb{Z}_{\geq 0}$ tal que $c(i) \neq c(j)$ para toda aresta $(i, j) \in E$ (ou, equivalentemente, $|c(i)-c(j)| \geq 1$ ) onde a quantidade de cores usadas seja a menor possível. Esse problema é um dos mais conhecidos em otimização combinatória e teoria dos grafos, com diversas aplicações, como alocação de canais em redes sem fio [Dias 2014, Koster 1999], escalonamento de tarefas [de Freitas et al. 2010], ensalamento escolar [Burke et al. 2010] e outros. Porém, o VCP é NP-difícil [Karp 1972], exigindo 
a utilização de técnicas avançadas para a obtenção de soluções ótimas ou próximas do ótimo em tempo computacional aceitável.

Diversos modelos de programação inteira (PI) foram propostos para o VCP, tais como: clássico baseado em restrições de atribuição, usado em método branch-and-cut [Méndez-Díaz and Zabala 2006]; baseado em conjuntos independentes maximais, utilizado em métodos de geração de colunas [Mehrotra and Trick 1996]; e formulação de representantes assimétricos, onde vértices são eleitos como representantes das classes de cores usadas [Campêlo et al. 2008].

Um outro modelo proposto é o baseado em orientação, proposto originalmente para a coloração em largura de banda (bandwidth coloring problem - BCP) [Dias et al. 2017], na qual as soluções induzem a uma orientação do grafo de entrada. Com base nesta formulação, deriva-se um modelo baseado em distâncias, onde, ao invés de determinar-se as cores explicitamente, as distâncias entre cores de vértices adjacentes devem ser retornadas, o que explora a correlação entre coloração de vértices e geometria de distâncias [Dias et al. 2013]. Sendo assim, neste trabalho, apresenta-se um estudo poliedral inicial dessa formulação baseada em distâncias, para a qual fornece-se duas famílias de desigualdades válidas indutoras de facetas do politopo associado.

\section{Formulação baseada em distância}

Dado um grafo $G=(V, E)$ e um conjunto de cores $C=\{1,2, \ldots,|V|\}$, o modelo baseado em distâncias utiliza variáveis inteiras $x_{i j}$ para todo $i, j \in V$ tal que $i<j$ que denotam a diferença entre as cores de $i$ e $j$ (de modo que $x_{i j}=c(i)-c(j)$ ). Além disso, também são usadas as variáveis de orientação $y_{i j}$, com valor 1 se $x_{i j}<0$ e 0 caso contrário. Uma solução factível para a coloração de vértices é dada pelas restrições a seguir:

$$
\begin{array}{ll}
x_{i k}=x_{i j}+x_{j k} & \forall i, j, k \in V, i<j<k \\
x_{i j} \geq 1-|C| y_{i j} & \forall(i, j) \in E, i<j \\
x_{i j} \leq-1+|C|\left(1-y_{i j}\right) & \forall(i, j) \in E, i<j \\
x_{i j} \in\{-|C|+1, \ldots,|C|-1\} & \forall i, j \in V, i<j \\
y_{i j} \in\{0,1\} & \forall(i, j) \in E, i<j
\end{array}
$$

As restrições (1) refletem a separação de cores entre os vértices, inclusive entre os não adjacentes entre si, de acordo com a interpretação das variáveis $x_{i j}$. Os conjuntos de restrições (2) e (3) induzem à orientação do grafo de acordo com as cores atribuídas. Por fim, (4) e (5) referem-se a integralidade e limites das variáveis.

O conjunto de restrições é composto de $O\left(|V|^{3}\right)$ equações, porém, o mesmo pode ser substituído por um conjunto de $O\left(|V|^{2}\right)$ equações, como visto a seguir.

Teorema 1 Se $V=\{1, \ldots, n\}$, então as restrições (1) equivalem a:

$$
\begin{array}{ll}
x_{i, i+1}+x_{i+1, i+2}=x_{i, i+2} & \forall i \in V, i \leq n-2 \\
x_{i j}+x_{i+1, j-1}=x_{i, j-1}+x_{i+1, j} & \forall i, j \in V, i \leq n-3, i+3 \leq j
\end{array}
$$

Seja $P D(G, C)$ o fecho convexo dos vetores $(x, y)$ que satisfazem as restrições (1)-(5). Para tal politopo, algumas propriedades do modelo são enunciadas, sendo a primeira relacionada à dimensão de $P D(G, C)$, como visto a seguir. 
Teorema $2 \mathrm{Se}|C|>\chi(G)+1$, então $\operatorname{dim}(P D(G, C))=|V|+|E|-1$.

Como dito anteriormente, o modelo baseado em orientação serve de base para esta formulação. Nele, existem variáveis inteiras $z_{i} \in\{1, \ldots,|C|\}$ para cada $i \in V$, além das mesmas variáveis $y$. Pode-se então transportar desigualdades válidas indutoras de facetas do politopo $\operatorname{PO}(G, C)$ do modelo com orientação para a formulação baseada em distâncias, como definido a seguir.

Teorema 3 Seja $\alpha z_{i}+\pi y \leq \alpha z_{j}+\pi_{0}$ uma desigualdade válida (indutora de faceta) para $P O(G, C)$, onde $(i, j) \in E$. Então, $\alpha x_{i j}+\pi y \leq \pi_{0}$ é válida (indutora de faceta se $|C| \geq \chi(G)+2)$ para $P D(G, C)$ e vice-versa.

\section{Facetas para modelo baseado em distâncias}

Uma importante implicação do Teorema 3 é que pode-se transformar desigualdades válidas indutoras de facetas do modelo baseado em orientação $(P O(G, C))$ para o modelo baseado em distâncias $(P D(G, C))$. A seguir, são mostradas duas famílias de desigualdades para $P O(G, C)$ e suas transformações, por meio do Teorema 3, para $P O(G, C)$. Ressalta-se que, nas expressões abaixo, o termo $z_{i}$ é a variável de $P O(G, C)$ que indica a cor do vértice $i \in V\left(\operatorname{com} z_{i} \in \mathbb{Z}_{\geq 0}\right)$. Também considere que $y_{j i}=1-y_{i j}$ para $i<j$.

\subsection{Desigualdade clique}

Seja $i \in V$ e $K \subseteq N(i)$ e uma clique. Define-se então a desigualdade clique associada a $i$ e $K$ para o $P O(G, C)$ do seguinte modo:

$$
z_{i} \geq \sum_{f \in K} y_{f i}
$$

Para utilizar a desigualdade no modelo baseado em distâncias pelo Teorema 3, deve-se adicionar um termo $z_{j}$ (onde $j$ é algum outro vértice diferente de $i$ ) para o lado direito da desigualdade, de modo a obter-se uma expressão $z_{i}-z_{j}$ a ser substituída por $x_{i j}$. Porém, deve-se adicionar um termo suficientemente grande também ao lado esquerdo para compensar tal adição. Desse modo, adiciona-se um termo $|C|$ do lado esquerdo, já que $z_{j} \in\{1,2, \ldots,|C|\}$. Assim, a desigualdade para $P D(G, C)$ é:

$$
x_{i j}+|C| \geq \sum_{f \in K} y_{f i}
$$

\subsection{Desigualdade clique dupla}

Seja $(i, j) \in E$ e considere uma clique $K \subseteq N(i) \cap N(j)$. Define-se então a desigualdade clique dupla associada ao vértice $i$ e à clique $K$ para $P O(G, C)$ como se segue:

$$
z_{i}+\sum_{k \in K}\left(y_{i k}-y_{j k}\right) \leq z_{j}+(s-|K|) y_{i j}
$$

Pela aplicação do Teorema 3 e pela observação que a constante $s$ do modelo baseado em orientação, que deve ser um limite superior para a maior cor usada, é equivalente a $|C|$ no modelo baseado em distâncias, obtém-se a desigualdade a seguir para $P D(G, C)$ :

$$
x_{i j}+\sum_{k \in K}\left(y_{i k}-y_{j k}\right) \leq(|C|-|K|) y_{i j}
$$




\begin{tabular}{|c|c|c|c|}
\hline Formulação & Variáveis & Restrições & Autor(es) \\
\hline Clássica & $O\left(|V|^{2}\right)$ & $O\left(|V|^{2} E\right)$ & - \\
\hline Conjuntos independentes & $O\left(3^{|V| / 3}\right)$ & $O(|V|)$ & [Mehrotra and Trick 1996] \\
\hline Representantes assimétricos & $O\left(|V|^{2}-|E|\right)$ & $O\left(|V|^{3}-|V||E|\right)$ & [Campêlo et al. 2008] \\
\hline Clique cover & $\stackrel{O}{O}(|V||H|)$ & $O\left(|H|+|V|\left|E^{\prime}\right|\right)$ & [Burke et al. 2010] \\
\hline $\begin{array}{c}\text { Baseada em orientação } \\
\text { Baseada em distância }\end{array}$ & $\begin{array}{c}O(|V|+|E|) \\
O\left(|V|^{2}\right)\end{array}$ & $\begin{array}{c}O(|\boldsymbol{V}|+|E|) \\
O\left(|V|^{2}\right)\end{array}$ & $\begin{array}{l}\text { [Dias et al. 2017] } \\
\text { Este trabalho }\end{array}$ \\
\hline
\end{tabular}

\section{Considerações finais}

Neste trabalho, apresentou-se uma formulação de programação inteira para o problema clássico de coloração de vértices em grafos (VCP), onde as variáveis de decisão indicam as distâncias entre cores dos vértices, desenvolvida com base em outra formulação de programação linear inteira proposta pelos autores, o modelo de orientação. Mostrouse que as desigualdades válidas indutoras de facetas do modelo baseado em orientação podem ser usadas na formulação com distâncias e vice-versa.

Resultados em andamento incluem a determinação de funções objetivo para o modelo baseado em distâncias, bem como a implementação de um método cut-and-branch utilizando a formulação e suas desigualdades válidas. Este trabalho também é a base para elaboração de um modelo de distâncias para a generalização do VCP conhecida como coloração em largura de banda (bandwidth coloring probem - BCP), que está em desenvolvimento.

\section{Referências}

Burke, E., Mareček, J., Parkes, A., and Rudová, H. (2010). A supernodal formulation of vertex colouring with applications in course timetabling. Annals of Operat. Research, 179:105-130.

Campêlo, M., Campos, V., and Corrêa, R. (2008). On the asymmetric representatives formulation for the vertex coloring problem. Discrete Applied Mathematics, 156:1097-1111.

de Freitas, R., Dourado, M., and Szwarcfiter, J. (2010). Graph coloring and scheduling problems. 4th Latin American Workshop on Cliques in Graphs.

Dias, B. (2014). Modelos teóricos e algoritmos para a otimização da alocação de canais em redes móveis sem fio. Master's thesis, Instituto de Computação - Universidade Federal do Amazonas, in portuguese.

Dias, B., de Freitas, R., Maculan, N., and Marenco, J. (2017). Facet-inducing inequalities and a cut-and-branch for the bandwidth coloring polytope based on the orientation model. Electronic Notes in Discrete Mathematics, 62:141 - 146. LAGOS'17 - IX Latin and American Algorithms, Graphs and Optimization.

Dias, B. R. C., Rodrigues, R. F., and Szwarcfiter, J. (2013). On graph coloring problems with distance constraints. In Proc. of I Workshop on Distance Geometry and Appl. (DGA 2013).

Karp, R. (1972). Reducibility Among Combinatorial Problems. In Miller, R. E. and Thatcher, J. W., editors, Complexity of Computer Computations, pages 85-103. Plenum Press.

Koster, A. (1999). Frequency assignment: models and algorithms. $\mathrm{PhD}$ thesis, Univ. Maastricht.

Mehrotra, A. and Trick, M. (1996). A column generation approach for graph coloring. INFORMS Journal on Computing, 8:344-354.

Méndez-Díaz, I. and Zabala, P. (2006). A branch-and-cut algorithm for graph coloring. Discrete Applied Mathematics, 154(5):826-847. 